\title{
Cinemática Posicional de Robôs via Iteração e Quatérnios
}

\author{
Luiz A. Radavelli , Daniel Martins \\ Depto de Engenharia Mecânica, Laboratório de Robótica, UFSC, \\ Campus Trindade \\ 88040-900, Florianópolis, SC \\ E-mail: luiz.radavelli@ufsc.br, daniel@emc.ufsc.br,
}

Edson R. De Pieri

Depto de Engenharia de Automação, UFSC

Campus Trindade

88040-900, Florianópolis, SC

E-mail: edopieri@das.ufsc.br,

\author{
Roberto Simoni \\ Depto de Engenharia da Mobilidade, UFSC \\ Campus Joinville \\ 89218-000, Joinville, SC \\ E-mail: roberto.simoni@ufsc.br.
}

\begin{abstract}
Resumo: Este trabalho resolve a cinemática posicional de robôs manipuladores utilizando um método iterativo proveniente da álgebra dos quatérnios e das convenções do método de DenavitHartenberg. Serão explicadas as condições necessárias para que as rotações sejam executadas por uma multiplicação simples de quatérnios ao invés do modo mais conhecido que utiliza uma multiplicação conjugada. O método iterativo apresentado utilizará a multiplicação simples de quatérnios, despendendo, portanto, menos operações que as abordagens tradicionais.
\end{abstract}

Palavras-chave: Quatérnios, Rotações, Cinemática, Iteração

\section{Introdução}

A cinemática posicional de robôs estuda a posição e orientação de robôs, relacionando os parâmetros das juntas atuadas com a posição e orientação do efetuador, abreviado por postura do efetuador. Os parâmetros das juntas atuadas são os ângulos de rotação e distâncias de translação das juntas. A cinemática posicional é dividida em dois subproblemas: cinemática direta, onde os parâmetros das juntas ativas são dados e estuda-se a postura do efetuador; e cinemática inversa, onde a postura do efetuador é conhecida e o problema consiste em determinar os parâmetros das juntas atuadas para que se obtenha tal postura.

Tradicionalmente, a cinemática posicional é obtida pelo método de Denavit-Hartenberg [12], mas a utilização de quatérnios e quatérnios duais neste contexto vem crescendo ao longo das últimas cinco décadas. As definições, operações e propriedades das álgebras dos quatérnios e dos quatérnios duais surgem de uma álgebra mais geral, a álgebra de Clifford [9].

Um dos primeiros trabalhos incluindo quatérnios na cinemática é atribuído a Yang e Freudenstein [17]. Depois dele vários trabalhos surgiram utilizando quatérnios e quatérnios duais. Na cinemática inversa [2], no problema das singularidades [14], na computação gráfica [13] e teoria de curvas [7], no planejamento de trajetória [4, 3], dinâmica [15]. Em [1] observam-se 
comparações entre métodos matriciais e quaternianos para resolver a cinemática posicional de robôs. Considerações computacionais relacionadas à utilização de quatérnios podem ser vistas em $[10,11]$. Para o estudo da cinemática posicional sugerimos [12]; para quatérnios $[5,8,6] \mathrm{e}$ $[9]$.

Baseando-se nas leituras citadas, principalmente em $[1,10,9]$, desenvolvemos a cinemática posicional de robôs utilizando a álgebra dos quatérnios e as convenções do método de DenavitHartenberg. Com os parâmetros de DH são definidos os operadores quatérnios de rotação que, por sua vez, resolvem a cinemática utilizando-se de um modelo computacional iterativo.

Para isto, o trabalho está organizado da seguinte forma: na Seção 2 são apresentados a álgebra dos quatérnios e o operador quatérnio de rotação. Ainda nesta seção são apresentadas uma correspondência matricial com os quatérnios que facilita a implementação da álgebra dos quatérnios, e uma forma mais econômica para as rotações. Na Seção 3 o método proposto é detalhado. Para exemplificar a aplicabilidade do método dois exemplos simples são apresentados. A Seção 4 foi reservada para algumas considerações finais.

\section{2 Álgebra dos Quatérnios e as Rotações}

Os quatérnios são elementos formados pela soma de escalares com vetores tridimensionais, aludindo-se à relação tempo × espaço. A parcela escalar de um quatérnio pode ser pensada como o eixo do tempo enquanto que a parcela vetorial, o espaço tridimensional [16]. O conjunto dos quatérnios encorpado de duas operações, adição e multiplicação, forma uma álgebra associativa, não comutativa, sem divisores de zero, denotada por $\mathbb{H}$, e que contém as álgebras $\mathbb{R}$ e $\mathbb{C}$.

Os quatérnios ganharam forma com Sir William Rowan Hamilton em 16 de outubro de 1843, quando ele descobrira a resposta para o problema que o atormentava: $i j=k$. Um quatérnio é um elemento abstrato que relaciona o tempo e o espaço, dado por

$$
q=w+x i+y j+z k=w+\mathbf{v}
$$

onde $w \in \mathbb{R}$ representa o eixo escalar, correspondente ao tempo, e $\mathbf{v}=\left[\begin{array}{ll}x & y \\ z\end{array}\right]^{T} \in \mathbb{R}^{3}$ representa o espaço. $i, j, k$ são as unidades quaternianas valendo-se das propriedades

$$
i^{2}=j^{2}=k^{2}=i j k=-1 \text {. }
$$

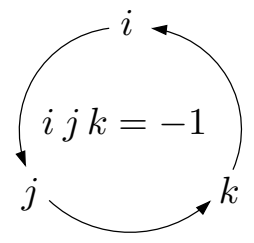

$S c(q):=w$ é dita a parte escalar (real) e $V e(q):=\mathbf{v}$ a parte vetorial (imaginária) do quatérnio $q$. Se $\mathbf{v}=0$, então $q=w$ é dito quatérnio real, enquanto que se $w=0$, então $q=\mathbf{v}$ é dito quatérnio puro. Neste caso o quatérnio corresponde aos vetores de $\mathbb{R}^{3}$. Observe que pelas propriedades supracitadas decorre que $i j=k, j k=i, k i=j, j i=-k, k j=-i$ e $i k=-j$.

Uma forma conveniente de representar um quatérnio é dada observando-se que um quatérnio $q \in \mathbb{H}$ pode ser associado a um elemento de $\mathbb{R}^{4}$, de modo que cria-se o seguinte mapeamento bijetivo:

$$
q=w+x i+y j+z k \in \mathbb{H} \longleftrightarrow q=\left[\begin{array}{llll}
w & x & y & z
\end{array}\right]^{T} \in \mathbb{R}^{4} .
$$

Além disso, a aplicação $\psi: \mathbb{H} \mapsto M_{4}(\mathbb{R})$ definida por

$$
\psi(w+x i+y j+z k)=\left[\begin{array}{cccc}
w & x & y & z \\
-x & w & -z & y \\
-y & z & w & -x \\
-z & -y & x & w
\end{array}\right]:=[q]_{\mathbb{R}}
$$

é um homomorfismo injetivo entre $\mathbb{H}$ e $M_{4}(\mathbb{R})$. Com essa representação e a definição da multiplicação de $\mathbb{H}$ (seção 2) fica fácil implementar a álgebra dos quatérnios. 


\section{1 Álgebra dos Quatérnios}

Considere $w_{k}, x_{k}, y_{k}, z_{k}$ números reais, $k=1,2$. Para os quatérnios

$$
\begin{aligned}
& q_{1}=w_{1}+x_{1} i+y_{1} j+z_{1} k=w_{1}+\mathbf{v}_{1}, \\
& q_{2}=w_{2}+x_{2} i+y_{2} j+z_{2} k=w_{2}+\mathbf{v}_{2},
\end{aligned}
$$

a álgebra dos quatérnios é estabelecida mediante as seguintes definições:

1. Igualdade: $q_{1}=q_{2} \Leftrightarrow w_{1}=w_{2}, x_{1}=x_{2}, y_{1}=y_{2}, z_{1}=z_{2}$.

2. Adição: $q_{1} \pm q_{2}=\left(w \pm w_{2}\right)+\left(\mathbf{v} \pm \mathbf{v}_{2}\right)$.

3. Multiplicação por escalar: $c q_{1}=c w+c \mathbf{v}, \quad \forall c \in \mathbb{R}$.

4. Multiplicação:

$$
\begin{aligned}
q_{1} q_{2}= & \left(w_{1}+x_{1} i+y_{1} j+z_{1} k\right)\left(w_{2}+x_{2} i+y_{2} j+z_{2} k\right) \\
= & w_{1} w_{2}-x_{1} x_{2}-y_{1} y_{2}-z_{1} z_{2} \\
& +\left(x_{1} w_{2}+w_{1} x_{2}-z_{1} y_{2}+y_{1} z_{2}\right) i \\
& +\left(y_{1} w_{2}+z_{1} x_{2}+w_{1} y_{2}-x_{1} z_{2}\right) j \\
& +\left(z_{1} w_{2}-y_{1} x_{2}+x_{1} y_{2}+w_{1} z_{2}\right) k .
\end{aligned}
$$

Computacionalmente, a multiplicação entre quatérnios pode ser definida utilizando-se o homomorfismo em (1). A saber, $q_{1} q_{2}=\left[q_{1}\right]_{\mathbb{R}}\left[q_{2}\right]_{\mathbb{R}}$ ou, também, $q_{1} q_{2}=\left[q_{1}\right]_{\mathbb{R}} q_{2}^{*}$. Não obstante, analisando-se os termos da multiplicação de quatérnios estabelece-se a forma vetorial, útil nos desenvolvimentos teóricos,

$$
q_{1} q_{2}=w_{1} w_{2}-\mathbf{v}_{1} \cdot \mathbf{v}_{2}+w_{1} \mathbf{v}_{2}+w_{2} \mathbf{v}_{1}+\mathbf{v}_{1} \times \mathbf{v}_{2} .
$$

Em particular, para quatérnios puros, $w_{1}=w_{2}=0 \mathrm{e}$

$$
\mathbf{v}_{1} \mathbf{v}_{2}=-\mathbf{v}_{1} \cdot \mathbf{v}_{2}+\mathbf{v}_{1} \times \mathbf{v}_{2},
$$

estabelece a relação entre o produto de quatérnios com os produtos interno e externo usuais de $\mathbb{R}^{3}$. Ainda para quatérnios puros,

$$
\begin{aligned}
& \mathbf{v}_{1} \mathbf{v}_{2}+\mathbf{v}_{2} \mathbf{v}_{1}=-2\left(\mathbf{v}_{1} \cdot \mathbf{v}_{2}\right), \\
& \mathbf{v}_{1} \mathbf{v}_{2}-\mathbf{v}_{2} \mathbf{v}_{1}=2\left(\mathbf{v}_{1} \times \mathbf{v}_{2}\right) .
\end{aligned}
$$

5. Conjugado: $q^{*}=w-\mathbf{v}$.

6. Norma: $\|q\|^{2}=q q^{*}=q^{*} q=w^{2}+x^{2}+y^{2}+z^{2} \in \mathbb{R}$. Um quatérnio é dito unitário se $\|q\|=1$.

7. Inverso: $q q^{-1}=q^{-1} q=1_{\mathbb{H}} \Leftrightarrow q^{*} q q^{-1}=q^{*} \Leftrightarrow\|q\|^{2} q^{-1}=q^{*} \Leftrightarrow q^{-1}=\frac{q^{*}}{\|q\|^{2}}$.

\subsection{Rotações por Quatérnios}

O grupo dos quatérnios unitários

$$
\mathrm{HU}(1)=\{q \in \mathbb{H} ;\|q\|=1\}
$$

constitui o conjunto dos quatérnios responsáveis por executar rotações em $\mathbb{R}^{3}$. De fato,

$$
q \in \mathrm{HU}(1) \Longleftrightarrow q=\cos \frac{\theta}{2}+\left(\operatorname{sen} \frac{\theta}{2}\right) \mathbf{s}
$$


onde $\mathbf{s}=\left[\begin{array}{lll}s_{x} & s_{y} & s_{z}\end{array}\right]^{T}$ é o vetor unitário que representa o eixo de rotação e $\theta$ a magnitude da rotação. Se $\mathbf{r} \in \mathbb{R}^{3}$ é codificado como um quatérnio puro, isto é, $\xi=0+\mathbf{r}$, então a rotação de $\mathbf{r}$ é dada pela multiplicação conjugada

$$
\xi^{\prime}=q \xi q^{*}
$$

Contudo, a rotação de $\mathbf{r}$ altera somente a componente de $\mathbf{r}$ que é perpendicular a $\mathbf{s}$ - veja a Figura 1. Em termos das componentes,

$$
\begin{aligned}
q \mathbf{r}_{\|} q^{*} & =\mathbf{r}_{\|}, \\
q \mathbf{r}_{\perp} q^{*} & =q^{2} \mathbf{r}_{\perp} .
\end{aligned}
$$

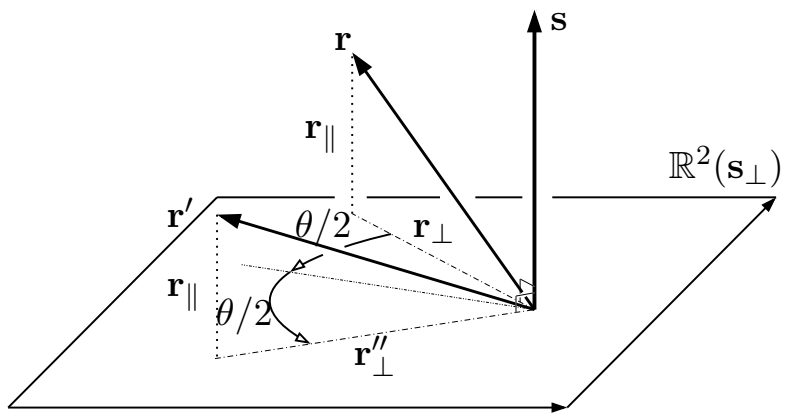

Figura 1: Ação da rotação de um vetor $\mathbf{r}$ via quatérnios.

Portanto, para $\mathbf{r} \perp \mathbf{s}$, a rotação via quatérnios pode ser estabelecida pela multiplicação simples

$$
\xi^{\prime}=q \xi, \quad \xi=0+\mathbf{r} .
$$

Porém neste caso o quatérnio responsável pela rotação é definido por

$$
q=\cos \theta+(\operatorname{sen} \theta) \mathbf{s}
$$

De fato, se $\mathbf{r} \perp \mathbf{s}$, então $\xi^{\prime}=q \xi q^{*}=q\left(0+\mathbf{r}_{\perp}\right) q^{*}=q^{2}\left(0+\mathbf{r}_{\perp}\right)$, em que

$$
q^{2}=\left[\cos \frac{\theta}{2}+\left(\operatorname{sen} \frac{\theta}{2}\right) \mathbf{s}\right]\left[\cos \frac{\theta}{2}+\left(\operatorname{sen} \frac{\theta}{2}\right) \mathbf{s}\right]=\cos \theta+(\operatorname{sen} \theta) \mathbf{s} .
$$

Observe que, conforme a Equação $(2), \mathbf{s}^{2}=\mathbf{s s}=-1$.

O método proposto contempla a propriedade $\mathbf{r} \perp \mathbf{s}$ em todas as ocasiões, valendo-se das rotações dadas por (5), com o quatérnio de rotação dado em (6).

\section{Cinemática Iterativa via Quatérnios}

Considere um robô manipulador composto por $n$ juntas rotativas ou prismáticas, modelado pela convenção de Denavit-Hartenberg. O método consiste em transformar os sistemas de coordenadas incorporados às componentes do robô de modo que a composição das transformações resulte na configuração final do efetuador, denotado por $P=(\mathcal{B}, \mathbf{r})$, em que $\mathcal{B}=O x y z=\{\mathbf{u}, \mathbf{v}, \mathbf{w}\}$, $\mathbf{u}=O x, \mathbf{v}=O y$ e $\mathbf{w}=O z$.

Basicamente, a transformação do frame $i-1$ para o frame $i$ é estabelecida seguindo-se as etapas [12] - veja a Fig. 2:

1. Rotação de $O x_{i-1}$ com magnitude $\theta_{i}$ em torno de $O z_{i-1}$;

2. Translação da origem $O_{i-1}$ com magnitude $d_{i}$ ao longo de $O z_{i-1}$;

3. Rotação de $O z_{i-1}$ com magnitude $\alpha_{i}$ em torno de $O x_{i}$; 


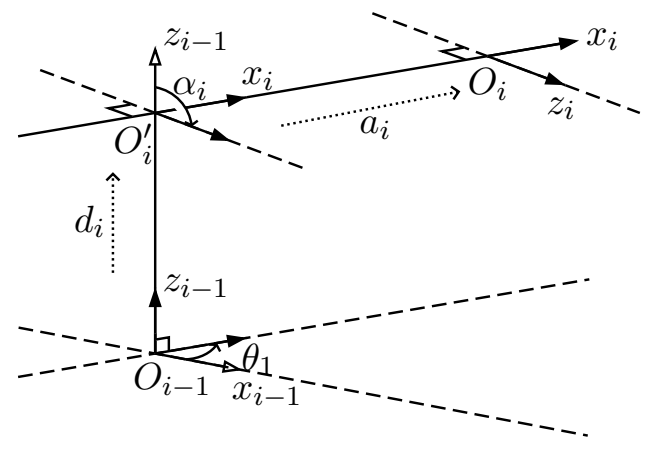

Figura 2: Esquema de deslocamento dos eixos que representam a configuração do robô.

4. Translação da origem $O_{i}^{\prime}$ com magnitude $a_{i}$ ao longo de $O x_{i}$.

A $n$-ésima iteração resultará no sistema de coordenadas do efetuador $\mathcal{B}$, isto é, $\mathbf{u}=\mathbf{x}_{n}$, $\mathbf{v}=\mathbf{y}_{n}$ e $\mathbf{w}=\mathbf{z}_{n}$. A origem de $\mathcal{B}$ revela a posição do efetuador, e é estabelecidas pela composição das etapas 2 e 4 .

A utilização de sistemas de coordenadas ortogonais garante que as etapas 1 e 3 sejam dadas por

$$
{ }^{x} \xi_{i}={ }^{z} q_{i}{ }^{x} \xi_{i-1} \quad \text { e } \quad{ }^{z} \xi_{i}={ }^{x} q_{i}{ }^{z} \xi_{i-1},
$$

onde ${ }^{x} \xi_{i}=0+\mathbf{x}_{i}, \quad{ }^{z} \xi_{i}=0+\mathbf{z}_{i}$ representam os eixos diretores dos sistemas de coordenadas $\mathcal{B}_{i}$ incorporados ao robô, e que ${ }^{z} q_{i}$ e ${ }^{x} q_{i}$ representam os operadores de rotação em torno dos eixos $O z$ e $O x$, respectivamente, definidos incorporando-se os parâmetros de $\mathrm{DH}$ em (6). Isto é:

$$
{ }^{z} q_{i}=\cos \theta_{i}+\left(\operatorname{sen} \theta_{i}\right) \mathbf{z}_{i-1} \quad \text { e }{ }^{x} q_{i}=\cos \alpha_{i}+\left(\operatorname{sen} \alpha_{i}\right) \mathbf{x}_{i} .
$$

Além disso, pela ortogonalidade entre os eixos dos sistemas de coordenadas, $\mathbf{y}_{i}=\mathbf{z}_{i} \times \mathbf{x}_{i}$.

Por fim, a composição das etapas 2 e 4 é dada pela iteração

$$
{ }^{r} \xi_{i}={ }^{r} \xi_{i-1}+d_{i}{ }^{z} \xi_{i-1}+a_{i}{ }^{x} \xi_{i},
$$

em que ${ }^{r} \xi_{i}=1+\mathbf{r}_{i}$ é o quatérnio que codifica o vetor posição $\mathbf{r}_{i}$. A $i$-ésima iteração resulta a configuração da $i$-ésima junta, $P_{i}=\left(\mathcal{B}_{i}, \mathbf{r}_{i}\right)$, enquanto que na $n$-ésima iteração $P=(\mathcal{B}, \mathbf{r})$ é revelado.

\subsection{Resultados}

Nesta seção foram utilizadas as abreviações: c $\theta=\cos \theta, \mathrm{s} \theta=\operatorname{sen} \theta, \theta i j=\theta_{i}+\theta_{j}$ e $\theta i \bar{j}=\theta_{i}-\theta_{j}$.

Considere o robô RRR planar. $R$ representa uma junta rotativa. Veja a Figura 3. Para ${ }^{x} \xi_{0}=\left[\begin{array}{llll}0 & 1 & 0 & 0\end{array}\right]^{T}$ e ${ }^{z} \xi_{0}=\left[\begin{array}{llll}0 & 0 & 0 & 1\end{array}\right]^{T}$, as iterações 1 , 2 e 3 levam às seguintes soluções:

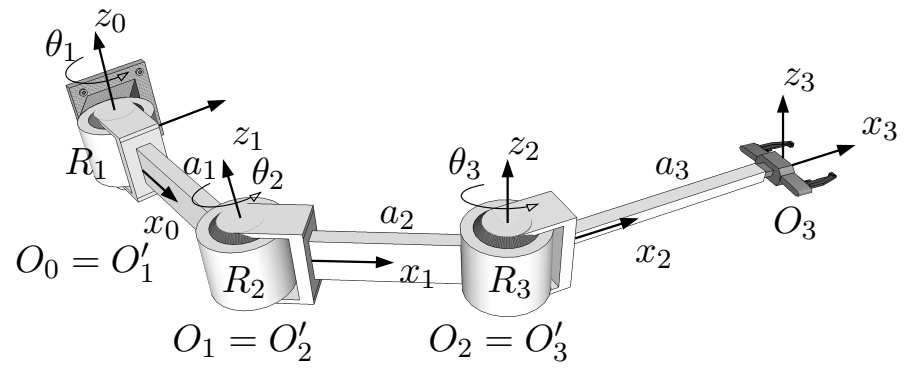

Figura 3: Robô RRR planar.

$$
{ }^{x} \xi_{1}=\left[\begin{array}{c}
0 \\
\mathrm{c} \theta_{1} \\
\mathrm{~s} \theta_{1} \\
0
\end{array}\right],{ }^{z} \xi_{1}=\left[\begin{array}{l}
0 \\
0 \\
0 \\
1
\end{array}\right], \quad{ }^{x} \xi_{2}=\left[\begin{array}{c}
0 \\
\mathrm{c} \theta_{12} \\
\mathrm{~s} \theta_{12} \\
0
\end{array}\right],{ }^{z} \xi_{2}=\left[\begin{array}{l}
0 \\
0 \\
0 \\
1
\end{array}\right], \quad{ }^{x} \xi_{3}=\left[\begin{array}{c}
0 \\
\mathrm{c} \theta_{123} \\
\mathrm{~s} \theta_{123} \\
0
\end{array}\right],{ }^{z} \xi_{3}=\left[\begin{array}{l}
0 \\
0 \\
0 \\
1
\end{array}\right] .
$$


O eixo $\mathbf{v}_{i}(i=1,2,3)$ é definido pelo produto externo: $\mathbf{v}_{i}={ }^{z} \xi_{i}(2: 4) \times{ }^{x} \xi_{i}(2: 4)$. Para a posição, as iterações retornam

$$
{ }^{r} \xi_{1}=\left[\begin{array}{c}
1 \\
a_{1} \mathrm{c} \theta_{1} \\
a_{1} \mathrm{~s} \theta_{1} \\
0
\end{array}\right], \quad{ }^{r} \xi_{2}=\left[\begin{array}{c}
1 \\
a_{1} \mathrm{c} \theta_{1}+a_{2} \mathrm{c} \theta_{12} \\
a_{1} \mathrm{~s} \theta_{1}+a_{2} \mathrm{~s} \theta_{12} \\
0
\end{array}\right], \quad{ }^{r} \xi_{3}=\left[\begin{array}{c}
1 \\
a_{1} \mathrm{c} \theta_{1}+a_{2} \mathrm{c} \theta_{12}+a_{3} \mathrm{c} \theta_{123} \\
a_{1} \mathrm{~s} \theta_{1}+a_{2} \mathrm{~s} \theta_{12}+a_{3} \mathrm{~s} \theta_{123} \\
0
\end{array}\right] .
$$

Observe que as iterações revelam as posições das juntas do robô.

Considere o robô SCARA. $R$ e $C$ representam juntas rotativas e cilíndricas, respectivamente. Veja a Figura 4. Para ${ }^{x} \xi_{0}=\left[\begin{array}{llll}0 & 1 & 0 & 0\end{array}\right]^{T} \mathrm{e}$ ${ }^{z} \xi_{0}=\left[\begin{array}{llll}0 & 0 & 0 & -1\end{array}\right]^{T}$, as iterações 1 , 2 e 3 levam às seguintes soluções:

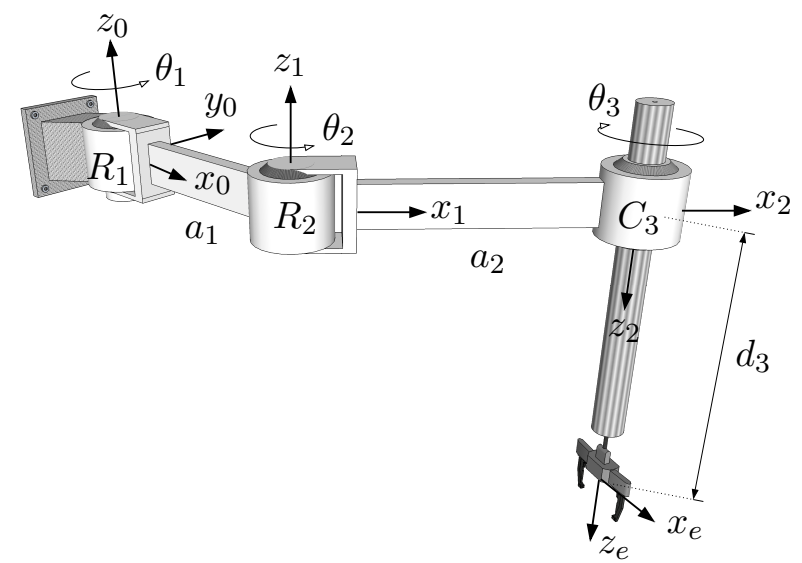

Figura 4: Robô RRC.

$$
{ }^{x} \xi_{1}=\left[\begin{array}{c}
0 \\
\mathrm{c} \theta_{1} \\
\mathrm{~s} \theta_{1} \\
0
\end{array}\right],{ }^{z} \xi_{1}=\left[\begin{array}{l}
0 \\
0 \\
0 \\
1
\end{array}\right], \quad{ }^{x} \xi_{2}=\left[\begin{array}{c}
0 \\
\mathrm{c} \theta_{12} \\
\mathrm{~s} \theta_{12} \\
0
\end{array}\right],{ }^{z} \xi_{2}=\left[\begin{array}{c}
0 \\
0 \\
0 \\
-1
\end{array}\right] \quad \text { e } \quad{ }^{x} \xi_{3}=\left[\begin{array}{c}
0 \\
\mathrm{c} \theta_{12 \overline{3}} \\
\mathrm{~s} \theta_{12 \overline{3}} \\
0
\end{array}\right],{ }^{z} \xi_{3}=\left[\begin{array}{c}
0 \\
0 \\
0 \\
-1
\end{array}\right] .
$$

O eixo $\mathbf{v}_{i}(i=1,2,3)$ é definido pelo produto externo: $\mathbf{v}_{i}={ }^{z} \xi_{i}(2: 4) \times{ }^{x} \xi_{i}(2: 4)$. Para a posição, as iterações retornam

$$
{ }^{r} \xi_{1}=\left[\begin{array}{c}
1 \\
a_{1} \mathrm{c} \theta_{1} \\
a_{1} \mathrm{~s} \theta_{1} \\
0
\end{array}\right], \quad{ }^{r} \xi_{2}=\left[\begin{array}{c}
1 \\
a_{1} \mathrm{c} \theta_{1}+a_{2} \mathrm{c} \theta_{12} \\
a_{1} \mathrm{~s} \theta_{1}+a_{2} \mathrm{~s} \theta_{12} \\
0
\end{array}\right] \quad \text { e } \quad{ }^{r} \xi_{3}=\left[\begin{array}{c}
1 \\
a_{1} \mathrm{c} \theta_{1}+a_{2} \mathrm{c} \theta_{12} \\
a_{1} \mathrm{~s} \theta_{1}+a_{2} \mathrm{~s} \theta_{12} \\
-d_{3}
\end{array}\right] .
$$

Observe que as iterações revelam as posições das juntas do robô. A última iteração resulta na posição do efetuador, codificada no quatérnio ${ }^{r} \xi_{3}$.

\section{Conclusões}

Este trabalho apresentou um método iterativo para resolver a cinemática posicional de robôs manipuladores. O método foi desenvolvido utilizando-se a álgebra dos quatérnios e os pressupostos da convenção de Denavit-Hartenberg. Também foi explicado em que condições as rotações via quatérnios podem ser executadas por multiplicação simples, $\xi^{\prime}=q \xi$, economizando um produto quaterniano em cada iteração, reduzindo o número de operações para a metade, quando comparado as rotações por multiplicação conjugada, $\xi^{\prime}=q \xi q^{*}$.

Quando comparado com o trabalho de Aspragathos e Dimitros, o método apresentado é computacionalmente ainda mais vantajoso, já que utiliza quatérnios ao invés de quatérnios duais para determinar a configuração de um robô.

Os exemplos, mesmo que simples, mostram a fácil aplicabilidade do método apresentado. Os problemas mais desafiadores serão os objetos das investigações futuras. 


\section{Referências}

[1] N. A. Aspragathos and J. K. Dimitros. A comparative study of three methods for robot kinematics. IEEE, 28(2):135-145, April 1998.

[2] Y. Aydin and S. Kucuk. Quaternion based inverse kinematics for industrial robot manipulators with euler wrist. IEEE, (1-4244-9713-4/06):581-586, 2006.

[3] R. Campa and K. Camarillo. Unit quaternions: A mathematical tool for modeling, path planning and control of robot manipulators. 2008.

[4] J. R. Dooley and J. M. McCarthy. On the geometric analysis of optimum trajectories for cooperating robots using dual quaternion coordinates. IEEE transactions on robotics and automation, pages 1031-1036, 1993.

[5] J. B. Kuipers. Quaternions and Rotation Sequences. Princeton University Press, 1999.

[6] P. Lounesto. Clifford Algebras and Spinors. Cambridge University Press, 2001.

[7] K. T. Miura. Unit quaternion integral curve: A new type of fair free-form curves. Computer Aided Geometric Design, 17:39-58, 2000.

[8] I. Porteous. Clifford algebra and the classical groups. Cambridge University Press, Cambridge, 1995.

[9] L. Radavelli. Análise cinemática direta de robôs manipuladores via álgebra de clifford e quatérnios. Dissertação, UFSC, 2013.

[10] L. Radavelli, R. Simoni, E. R. De Pieri, and D. Martins. A comparative study of the kinematics of robot manipulators by Denavit-Hartenberg and dual quaternion. Mec $\tilde{A}$;nica Computacional, Vol.XXXI:pp.2833-2848, Noviembre 2012.

[11] S. Sahul, B. B. Biswall, and B. Subudhi. A novel method for representing robot kinematics using quaternion theory. IEEE sponsored conference on computational intelligence, control and computer vision in robotics 83 automation, 2008.

[12] L. Sciavicco, L. Villani, G. Oriolo, and B. Siciliano. Robotics: Modelling, planning and control. Springer-Verlag, London, 2009.

[13] K. Shoemake. Quaternion Calculus for Animation. SIGGRAPH '89, 1989.

[14] M. Shoham and P. Ben-Horin. Application of grassmann-cayley algebra to geometrical interpretation of parallel robot singularities. Int. J. Rob. Res., 28:127-141, 2009.

[15] J.-Y. Wang, H.-Z. Liang, Z.-W. Sun, S.-N. Wu, and S.-J. Zhang. Relative motion coupled control based on dual quaternion. Aerospace Science and Technology, 25:102-113, 32013.

[16] J. P. Ward. Quaternions and Cayley Numbers, volume 403. Springer Science Business Media Dordrecht, 1997.

[17] A. T. Yang and F. Freudenstein. Application of dual-number quaternions to the analysis of the spatial mechanism. AMSE Transactions Journal of Applied Mchanics, 86:300-308, June 1964. 